\title{
Efficacy and Adverse Effects of Transpulmonary Contrast Echocardiography Using Sonicated Albumin
}

\author{
Satoshi Nakatani, Shintaro Beppu*, Takahiro OhtA*, Hisao Matsuda* and Kunio Miyatake
}

\begin{abstract}
We studied the feasibility and adverse effects of transpulmonary contrast echocardiography using sonicated albumin in 6 dogs. Left heart contrast was observed by two-dimensional echocardiography, monitoring left ventricular pressure, its first derivative $(\mathrm{dP} / \mathrm{dt})$ and arterial oxygen saturation. The left heart was adequately opacified in 48 of 55 injections of various intravenous doses of sonicated albumin. Left ventricular myocardial opacification was faintly observed in only 1 injection. After injections of a large dose $(0.5 \mathrm{ml} / \mathrm{kg})$, left ventricular systolic pressure decreased, maximal positive $\mathrm{dP} / \mathrm{dt}$ decreased and negative $\mathrm{dP} / \mathrm{dt}$ increased, slightly. Hemodynamic parameters showed no significant changes with the other doses. Arterial oxygen saturation tended to decrease after injection, which was most remarkable with a large dose injection. In conclusion, although a large dose of intravenous sonicated albumin may opacify not only the left heart cavity but the ventricular myocardium, it deteriorates hemodynamic parameters and oxygen exchange in the lung.
\end{abstract}

(Internal Medicine 33: 204-209, 1994)

Key words: ultrasound, contrast medium, microbubbles, cardiac function, arterial oxygen saturation

\section{Introduction}

Left heart contrast echocardiography has been performed by injecting contrast agents directly into the aortic root $(1,2)$, the left ventricle $(3,4)$ or the coronary artery $(2,5-8)$. Although this technique has been helpful to identify abnormal cardiac structure $(1,3)$, determine valvular regurgitation (4), demonstrate left-to-right shunts $(4,9)$ and evaluate myocardial perfusion $(2$, 5-8), the insertion of arterial catheters was required.

Recently, several investigators have reported left heart contrast echocardiography following intrapulmonary, right atrial or peripheral venous injection of contrast agents capable of transpulmonary passage (10-16). It is anticipated that if a large dose of a transpulmonary contrast agent is injected intravenously, enough microbubbles would reach the left heart cavity to opacify the left ventricular myocardium. Before applying this technique to patients, however, possible complications attributable to a large dose administration should be recognized.

Among transpulmonary contrast agents, sonicated albumin has been proposed as a more ideal contrast agent because its microbubbles are relatively small and cause neither a hyperemic response nor significant hemodynamic effects even with intracoronary use $(17,18)$. Although custom-made sonicated albumin microspheres (Albunex, Molecular Biosystems Inc., San Diego, CA., U.S.A.) have been recently developed, further clinical trials are required before its approval for human use (10, 11). Therefore, in the present study, we made albumin microbubbles by sonication and investigated the feasibility of transpulmonary contrast echocardiography and the adverse effects of this contrast agent.

\section{Material and Methods}

\section{Contrast agent preparation}

Fifteen $\mathrm{ml}$ of $5 \%$ human serum albumin solution in a $20 \mathrm{ml}$ plastic syringe was sonicated with a commercially available sonicating system (Sonifier 250, Branson Inc., Danbery, CT., U.S.A.) using a $3 \mathrm{~mm}$ titanium-tipped horn at $20 \mathrm{kHz}$ at a power output of $20 \mathrm{~W}$ for 30 seconds (19). The tip of the horn was placed just below the surface of the solution and was fixed at that position throughout the sonication. Immediately after sonication, the solution was drained from the bottom of the syringe into a separate syringe and used for injection after discarding the first

From the Cardiology Division and Research Institute* and National Cardiovascular Center, Suita

Received for publication August 23, 1993; Accepted for publication December 27, 1993

Reprint requests should be addressed to Dr. Satoshi Nakatani, the Department of Cardiology, Desk F15, The Cleveland Clinic Foundation, 9500 Euclid Avenue, Cleveland, OH 44195, U.S.A. 
$1 \mathrm{ml}$. The median size of albumin microbubbles constituting this solution was $5 \mu \mathrm{m}$ (weight mean $\pm \mathrm{SD}, 11 \pm 12 \mu \mathrm{m}$ ) and the number was 391,000 per $\mathrm{ml}$, mostly consisting of small microbubbles less than $10 \mu \mathrm{m}$ as measured by a Coulter counter (Model B, Coulter Electronics, Hialeah, FLA., U.S.A.) as previously reported (19).

\section{Experimental preparation}

Six mongrel dogs, weighing from 12 to $20 \mathrm{~kg}$, were anesthetized with intravenous administration of $30 \mathrm{mg} / \mathrm{kg}$ sodium pentobarbital, intubated and provided with respiration by a positive pressure respirator (Bird Mark 7, Palm Springs, CA., U.S.A.) with a mixture of room air and oxygen. Arterial oxygen saturation was maintained between 87 and $93 \%$ by controlling the fraction of inspiratory oxygen and the tidal volume to simulate patients with impaired lung function. A median thoracotomy was performed. Administration of saline and drugs was performed through a plastic cannula $(2 \mathrm{~mm}$ in diameter) inserted into the femoral vein. Drip infusion of saline solution throughout the experiment was at a rate of $100 \mathrm{ml} / \mathrm{hr}$. A 5F micromanometer-tip catheter (Millar Mikro-tip, model PC-350, Millar Instruments, Inc., Houston, TX., U.S.A.) was inserted into the left ventricle via the right femoral artery to measure left ventricular systolic and end-diastolic pressures and the first derivative of left ventricular pressure $(\mathrm{dP} / \mathrm{dt})$. A 4F fiberoptic catheter (Opticath U440, Abbot Critical Care Systems, Mountain View, CA.) was inserted into the left femoral artery and arterial oxygen saturation was continuously measured with an Oximetrix (Oximetrix 3, Abbot Critical Care Systems). The accuracy of this catheter oximetry system has been previously validated $(20,21)$.

\section{Experimental protocol}

Various doses of sonicated albumin were randomly bolus injected into the femoral vein and followed immediately by a 5 $\mathrm{ml}$ saline flush. The doses were 0.025 ( 16 trials), 0.05 (18 trials), 0.25 ( 8 trials) and $0.5 \mathrm{ml} / \mathrm{kg}$ (13 trials). After each injection, there was a lapse of at least 7 minutes before the next injection. Two-dimensional and M-mode echocardiograms were obtained by a commercially available $5 \mathrm{MHz}$ phased-array transducer with an echocardiograph system (Toshiba SSH-60A, Toshiba Medical, Tokyo, Japan) during each injection and were recorded on a half-inch VHS videotape recorder (Model 6400, Victor, Tokyo, Japan). To avoid echo attenuation in the left heart due to strong contrast echo in the right heart, four chamber views were obtained by placing the transducer directly on the left ventricle. Since a dog has a vertical heart, it was difficult to put the transducer just on the apex in our model and thus, a distorted four chamber view was obtained (Fig. 1). Gray scale, depth, power, and reject or dynamic range controls were adjusted to provide the best ultrasound image during the baseline recordings and were held constant for the experiment in each dog. M-mode echocardiograms of the left heart were also recorded on a strip chart recorder at a paper speed of $25 \mathrm{~mm} / \mathrm{sec}$. A standard lead II electrocardiogram, left ventricular pressure, $\mathrm{dP} / \mathrm{dt}$ and arterial oxygen saturation were recorded continu- ously with a multichannel recorder (Nihon Denki San-ei, Tokyo, Japan) during each injection. All of the recordings and measurements were performed without turning off the respirator. The animals used in this study were handled in accordance with the guidelines of the committee on animal studies at the National Cardiovascular Center.

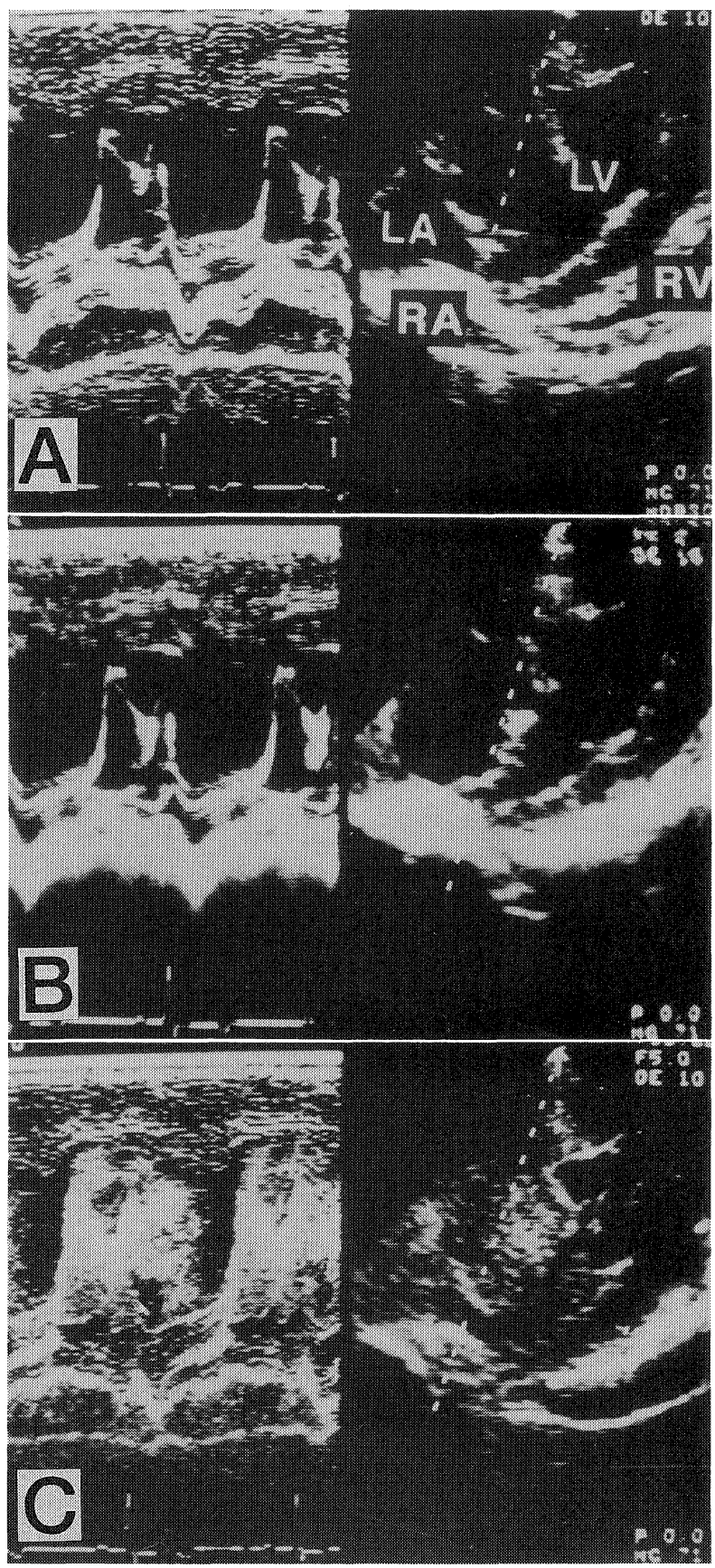

Fig. 1. Two-dimensional (right column) and M-mode (left column) echocardiograms before and after intravenous $0.5 \mathrm{ml} / \mathrm{kg}$ sonicated albumin injection. A) before injection. B) 2 seconds after injection. The right atrium and right ventricle are opacified. C) 5 seconds after injection. The left atrium and ventricle are also opacified. LA: left atrium, LV: left ventricle, RA: right atrium, RV: right ventricle. 


\section{Analysis of contrast intensity}

To quantify the contrast echo intensity, we used a commercially available high-speed image processing system capable of digitizing echocardiographic fields in real time (Model LA555, PIAS Co, Tokyo, Japan) using a personal computer (Model PC-9801, NEC Co, Tokyo, Japan). This system was used to convert each two-dimensional echocardiographic image in the video tape to a $512 \times 512$ pixel image with 256 gray levels/pixel and to quantify the intensity of echocardiographic signals in the regions of interest that were outlined by the operator. We positioned the regions of interest in the center of the left ventricle and the interventricular septum excluding the endocardium and epicardium. The regions of interest were circles with a diameter of $8 \mathrm{~mm}$ and $5 \mathrm{~mm}$, respectively. When outlining the regions in the ventricular cavity, great care was taken to avoid cardiac structures such as myocardial walls, mitral and tricuspid chordae and valve leaflets irrespective of the respiratory change of cardiac position. The gray level within the region of interest before injection was subtracted from the values after injection. The subtracted values (contrast echo intensities) were printed with the frame numbers. The system used also printed the plot of contrast echo intensities in relation to time from the first analyzed end-diastole. In the analysis of the ventricular cavity opacification, peak contrast intensities and durations of opacification showing more than 5 units of contrast intensity were analyzed.

\section{Statistical analysis}

All data are expressed as mean \pm SD. Paired t test was used to compare hemodynamic data and arterial oxygen saturation before and after injection of sonicated albumin. Peak contrast intensity and duration were compared among groups by means of analysis of variance and Scheffe's F test. A p value less than 0.05 was considered significant.

\section{Results}

\section{Left heart opacification}

We intravenously injected sonicated albumin 55 times in total. In 48 trials, the left heart cavity was adequately opacified after several beats of right heart opacification (Fig. 1). From the analysis of the time-contrast intensity curve, the peak contrast intensity tended to be higher and the duration of opacification tended to be longer as the injected dose increased (Figs. 2, 3). Left ventricular myocardial opacification was faintly visualized in only 1 of 13 injections at a large dose of sonicated albumin $(0.5 \mathrm{ml} / \mathrm{kg})$. Time-contrast echo intensity curves in the regions of the left ventricular cavity and the interventricular septum of that case shows myocardial opacification following left heart cavity opacification (Fig. 4). Contrast enhancement of the left ventricular myocardium was not seen with the other trials.

Hemodynamic data and oxygen saturation (Table 1, Fig. 5)

Hemodynamic parameters did not show significant changes after intravenous injection of 0.025 and $0.05 \mathrm{ml} / \mathrm{kg}$ sonicated

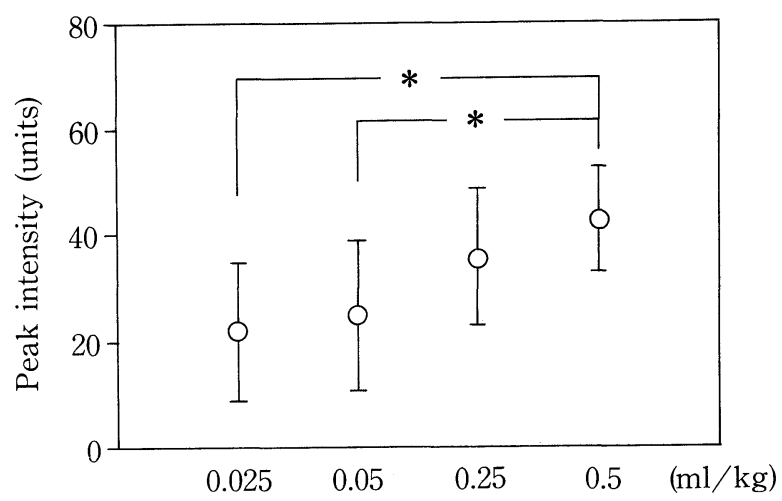

Fig. 2. Relation of injected sonicated albumin doses and peak contrast intensity. The peak contrast intensity tends to be higher as the injected dose increases. The vertical lines with an open circle represent the mean value $\pm 1 \mathrm{SD}$. $* \mathrm{p}<0.05$.

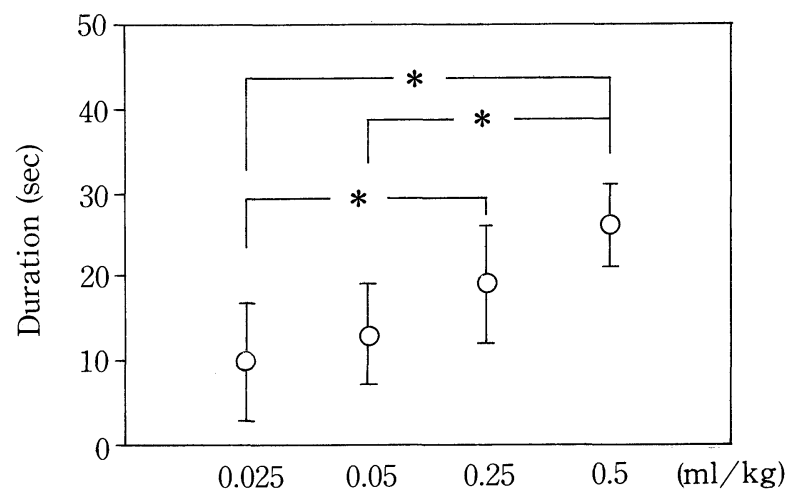

Fig. 3. Relation of injected sonicated albumin doses and the duration of the opacification showing more than 5 units of contrast intensity. The duration tends to be longer as the injected dose increases. The vertical lines with an open circle represent the mean value $\pm 1 \mathrm{SD} .{ }^{*} \mathrm{p}<0.05$.

albumin. With the $0.25 \mathrm{ml} / \mathrm{kg}$ injection, only the left ventricular systolic pressure decreased slightly. In contrast, after injections at a large dose of $0.5 \mathrm{ml} / \mathrm{kg}$, left ventricular systolic pressure decreased and the amplitude of maximal positive and negative $\mathrm{dP} / \mathrm{dt}$ decreased, although the changes were transient (Fig. 5). Left ventricular end-diastolic pressure did not show significant changes with all injections. Arterial oxygen saturation tended to decrease after intravenous sonicated albumin. The decrease was most remarkable with a large dose of $0.5 \mathrm{ml} / \mathrm{kg}$ (mean $3 \%$ ). As shown in Figure 5, arterial oxygen saturation decreased significantly for about 30 seconds after injection and it required about 1 to 2 minutes to recover completely.

\section{Discussion}

\section{Left heart cavity opacification}

Keller and colleagues have demonstrated that sonicated albumin was an echogenic agent capable of transpulmonary passage (12). It was shown that 3 to $5 \mathrm{ml}$ of intravenous 

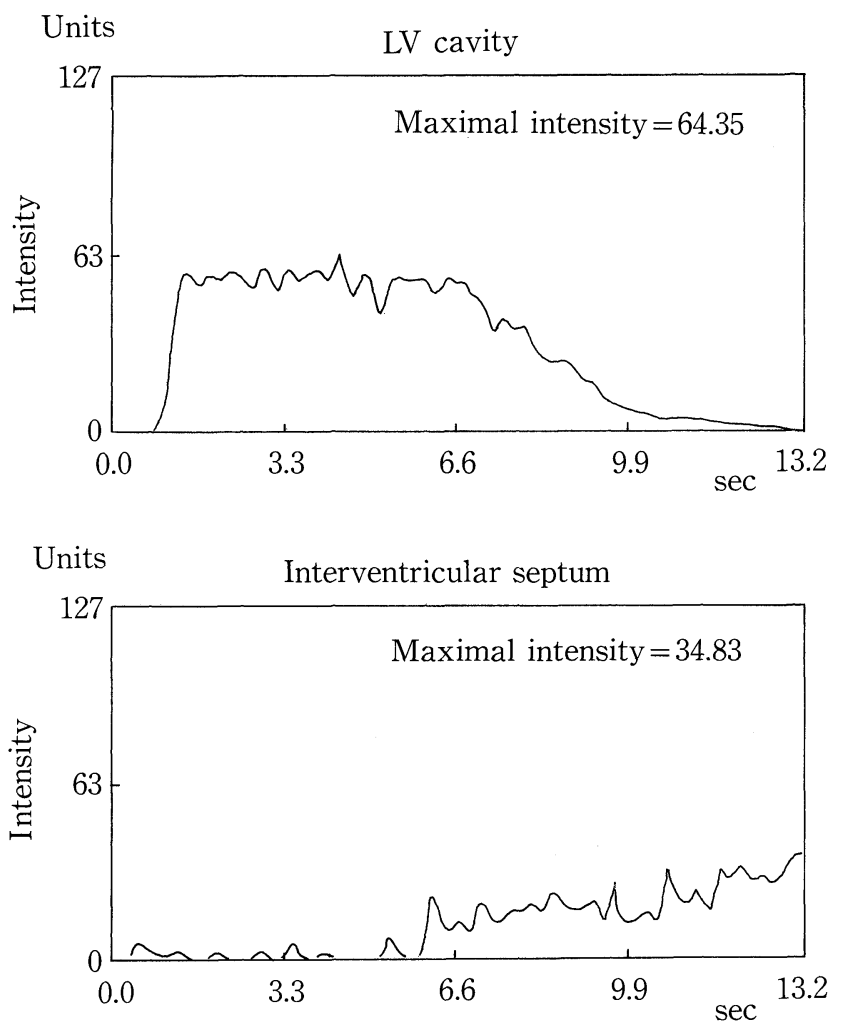

Fig. 4. Time-contrast intensity curves in the left ventricular (LV) cavity (upper panel) and in the interventricular septum (lower panel) in one dog which showed left ventricular myocardial opacification after intravenous injection of $0.5 \mathrm{ml} / \mathrm{kg}$ sonicated albumin. The interventricular septum is opacified following left ventricular cavity opacification.

sonicated albumin could demonstrate contrast enhancement of the left ventricular cavity of dogs. We also have shown that the left heart cavity was opacified by intravenous sonicated albumin even in a smaller dose than they used. Moreover, we showed that the larger the injected dose, the more intense the opacification and the longer the duration of the contrast effect. Previously, some attempts were made to estimate cardiac output (13) or the severity of aortic regurgitation (14) using transpulmonary contrast echo agents. Others have advocated that transpulmonary contrast echo agents might allow more sensitive diagnosis of left-to-right shunts (15), better imaging of the left heart structures $(15,16)$ and estimation of left ventricular ejection fraction (22). Furthermore, successful attaining of contrast agents in the left heart cavity would be useful in enhancing left heart Doppler signals (23-25). Adequate and long-term left heart opacifications are desirable to achieve these objectives. Therefore, knowledge of the dose dependency of the contrast effect, which we have shown here, may provide better clinical applications of this technique.

\section{Left ventricular myocardial perfusion imaging}

There have been many reports that demonstrate the ability of myocardial perfusion imaging by contrast-enhanced two-dimensional echocardiography $(2,5-8)$. They injected contrast agents either into the aortic root or directly into the coronary arteries and few have tried to demonstrate myocardial perfusion imaging by transpulmonary contrast agents. In the current study, myocardial opacification was faintly visualized in 1 injection with a large dose, which was confirmed using a highspeed image processing system. However, the myocardial opacification was not reproducible in the other 11 injections with the same dose. Therefore, it is considered that myocardial perfusion imaging with a peripheral injection of sonicated albumin must be difficult in the present experimental system. Ten Cate et al reported that they observed left ventricular myocardial opacification in 24 of 152 studies (16\%) of intrapulmonary injections of $8 \mathrm{ml}$ of sonicated Renografin-76 (Schering Japan Co., Osaka, Japan) or sorbitol 70\% (13). On the other hand, Smith and coworkers reported that they did not observe the left ventricular myocardial opacification after intravenous injection of $2 \mathrm{ml}$ of the saccharide lung-crossing agent SHU-508 (Schering Japan Co., Osaka, Japan) (16). Recently,

Table 1. Hemodynamic Parameters and Arterial Oxygen Saturation before and after Intravenous Sonicated Albumin

\begin{tabular}{|c|c|c|c|c|c|c|}
\hline \multicolumn{2}{|c|}{ Dose (ml/kg) } & \multirow{2}{*}{$\frac{\operatorname{LVSP}(\mathrm{mmHg})}{132 \pm 25}$} & \multirow{2}{*}{$\frac{\operatorname{LVEDP}(\mathrm{mmHg})}{6 \pm 3}$} & \multirow{2}{*}{$\frac{\mathrm{dP} / \mathrm{dt}(\mathrm{mmHg} / \mathrm{sec})}{909 \pm 51}$} & \multirow{2}{*}{$\frac{-\mathrm{dP} / \mathrm{dt}(\mathrm{mmHg} / \mathrm{sec})}{-1292 \pm 268}$} & \multirow{2}{*}{$\frac{\mathrm{SaO} 2(\%)}{90.7 \pm 0.9}$} \\
\hline 0.025 & before & & & & & \\
\hline$(\mathrm{n}=12)$ & after & $132 \pm 25$ & $6 \pm 3$ & $888 \pm 53$ & $-1292 \pm 268$ & $90.6 \pm 1.0$ \\
\hline 0.05 & before & $131 \pm 24$ & $6 \pm 2$ & $833 \pm 51$ & $-1237 \pm 242$ & $89.4 \pm 3.2$ \\
\hline$(n=15)$ & after & $130 \pm 24$ & $5 \pm 2$ & $813 \pm 62$ & $-1203 \pm 229$ & $88.9 \pm 3.3^{*}$ \\
\hline 0.25 & before & $128 \pm 23$ & $6 \pm 2$ & $881 \pm 70$ & $-1256 \pm 269$ & $88.5 \pm 2.6$ \\
\hline$(n=8)$ & after & $125 \pm 24 *$ & $5 \pm 1$ & $850 \pm 60$ & $-1225 \pm 254$ & $87.4 \pm 2.9$ \\
\hline 0.5 & before & $126 \pm 16$ & $6 \pm 2$ & $906 \pm 87$ & $-1308 \pm 188$ & $87.8 \pm 2.5$ \\
\hline$(\mathrm{n}=13)$ & after & $117 \pm 18^{*}$ & $6 \pm 2$ & $849 \pm 91 *$ & $-1204 \pm 213 *$ & $84.9 \pm 4.2 *$ \\
\hline
\end{tabular}

Values are expressed as mean values $\pm \mathrm{SD}$. ${ }^{*} \mathrm{p}<0.01$ versus before. LVSP: left ventricular systolic pressure, LVEDP: left ventricular end-diastolic pressure, $\mathrm{dP} / \mathrm{dt}$ : maximal value of the first derivative of left ventricular pressure, - $\mathrm{dP} /$ $\mathrm{dt}$ : minimal value of the first derivative of left ventricular pressure, $\mathrm{SaO} 2$ : arterial oxygen saturation. 


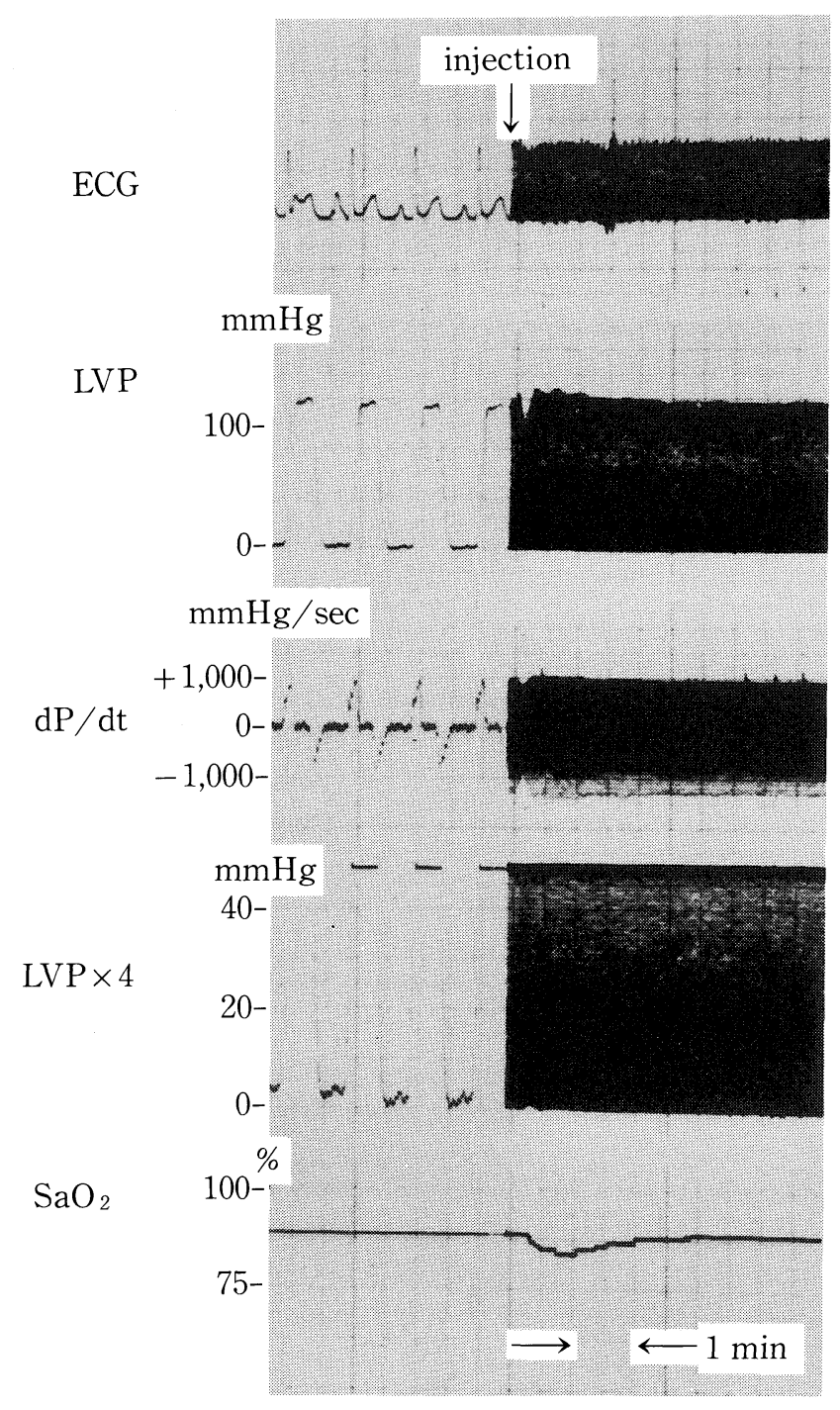

Fig. 5. Recordings showing effects of intravenous injection of $0.5 \mathrm{ml} / \mathrm{kg}$ sonicated albumin on hemodynamic parameters and arterial oxygen saturation. Left ventricular systolic pressure and maximal derivative of left ventricular pressure transiently decreases and minimal derivative of left ventricular pressure transiently increases after injection. Note that arterial oxygen saturation decreased by $5 \%$ about 30 seconds after injection and the decrease continues for about 1 to 2 minutes. This recording after injection was done at a paper speed of $1 \mathrm{~cm} / \mathrm{min}$ for easiness of understanding. $\mathrm{dP} / \mathrm{dt}$ : first derivative of left ventricular pressure, ECG: electrocardiogram, LVP: left ventricular pressure, $\mathrm{LVP} \times 4$ : magnifying by four of left ventricular diastolic pressure tracing, $\mathrm{SaO} 2$ : arterial oxygen saturation.

Albunex has been shown to opacify the left ventricular myocardium successfully and reproducibly after intravenous or right atrial injection (10). The difference of success rates of myocardial opacification may be explained mainly by the differences of dose and size of microbubbles.

\section{Adverse effects of intravenous sonicated albumin}

To date, most reports have not demonstrated any serious adverse effect of intravenous or intrapulmonary injection of contrast agents $(11-13,15,16)$. Some investigators assessed the effect of intravenous contrast agents on arterial blood gas by blood sampling $(12,16)$. However, they might not detect an acute or temporary change in arterial blood gas induced by contrast agents because they did not monitor the change continuously. To continuously measure arterial oxygen saturation, we used an oximeter catheter and studied the effects of various doses of sonicated albumin on arterial blood gas. We found a transient but significant decrease in arterial oxygen saturation after a moderate to large dose of sonicated albumin injection, which soon recovered. There have been a few reports that demonstrated hemodynamic deterioration attributable to intravenous injection of contrast agents $(14,26)$. We, unlike others $(11-13,15,16)$, have found transient but significant deteriorations in hemodynamic parameters after a large dose injection, which also soon recovered without special treatment.

The cause of these adverse effects are unknown. Although we preliminarily checked the effect of a large dose of intravenous non-sonicated albumin $(0.5 \mathrm{ml} / \mathrm{kg})$ on hemodynamics and arterial oxygen saturation, no significant adverse effects were observed. Therefore, adverse effects described here may not be due to a large dose of albumin itself. The median size of the albumin microbubble used in the present study was as small as $5 \mu \mathrm{m}$, but larger microbubbles were contained in the injected solution. They might cause temporary obstruction of pulmonary capillary flow and might lead to deterioration of arterial oxygen saturation. A recent study has shown that intravenous injection of Albunex (microbubble size, $3.9 \pm 0.3$ to $5.8 \pm 0.1$ $\mu \mathrm{m})$, even with a larger dose than the present study's, did not deteriorate arterial oxygen tension (27). It also has been demonstrated that Albunex did not induce any changes in the left atrial, left ventricular and pulmonary artery pressures after injection to the right atrium $(11,27)$. These studies support our hypothesis that the microbubble size is an important factor that influences gas exchange in the lung and hemodynamics. Whether these changes in hemodynamics and gas exchange in the lung were based on organ ischemia or not remain unclear. More studies are needed concerning the mechanism of these adverse effects.

\section{Clinical implications}

We have demonstrated the efficacy and limitations of intravenous sonicated albumin as a transpulmonary echo-contrast agent. Intravenous sonicated albumin can adequately opacify the left heart cavity even in a small dose of $0.025 \mathrm{ml} / \mathrm{kg}$ without adverse effects. Theoretically, myocardial perfusion imaging with peripheral venous injection of contrast agents may be accomplished if adequate contrast effect is achieved in the left heart cavity. However, we showed that when a large dose of sonicated albumin was injected, hemodynamic parameters and arterial oxygen saturation might deteriorate. Although these changes soon recovered without special treatment, the present results might provide caution for the clinical application of a large dose of intravenous sonicated albumin to patients who have impaired systolic and/or arterial gas exchange function. However, the present study does not degrade the usefulness of transpulmonary contrast echocardiography. It is important that 


\section{Transpulmonary Contrast Echo by Albumin}

a small dose of sonicated albumin could enhance the left heart cavity without any adverse effects. Moreover, the adverse effects induced by a large dose of sonicated albumin will be mitigated by the use of nontoxic, stable and smaller size contrast agents such as Albunex $(11,27)$. Transpulmonary contrast echocardiography is an exciting technique for the future. Further development of contrast agents and easy analysis methods will be needed before this technique can be widely used clinically.

\section{References}

1) Gramiak R, Shah PM. Echocardiography of the aortic root. Investigative Radiol 3: 356, 1968.

2) Armstrong WF, West SR, Mueller TM, Dillon JC, Feigenbaum H. Assessment of location and size of myocardial infarction with contrastenhanced echocardiography. J Am Coll Cardiol 2: 63, 1983.

3) Sahn DJ, Williams DE, Shackelton S, Friedman WF. The validity of structure identification for cross-sectional echocardiography. J Clin Ultrasound 3: 201, 1974.

4) Kerber RE, Kioschos JM, Laver RM. Use of ultrasonic contrast method in the diagnosis of valvular regurgitation and intracardiac shunts. Am J Cardiol 34: 722, 1974.

5) Tei C, Kondo S, Meerbaum S, et al. Correlation of myocardial echo contrast disappearance rate ("washout") and severity of experimental coronary stenosis. J Am Coll Cardiol 3: 39, 1984.

6) Kaul S, Glasheen W, Ruddy TD, Pandian NG, Weyman AE, Okada RD. The importance of defining left ventricular area at risk in vivo during acute myocardial infarction: an experimental evaluation with myocardial contrast two-dimensional echocardiography. Circulation 75: 1249, 1987.

7) Feinstein SB, Lang RM, Dick C, et al. Contrast echocardiography during coronary arteriography in humans: perfusion and anatomic studies. J Am Coll Cardiol 11: 59, 1988.

8) Reisner SA, Ong LS, Lichtenberg GS, et al. Quantitative assessment of the immediate results of coronary angioplasty by myocardial contrast echocardiography. J Am Coll Cardiol 13: 852, 1989.

9) Pieroni DR, Varghese J, Freedom RM, Rove RD. The sensitivity of contrast echocardiography in detecting intracardiac shunts. Cathet Cardiovasc Diagn 5: 19, 1979.

10) Sanders WE, Cheirif J, Desir R, et al. Contrast opacification of left ventricular myocardium following intravenous administration of sonicated microspheres. Am Heart J 122: 1660, 1991.

11) Villanueva FS, Glasheen WP, Sklenar J, Jayaweera AR, Kaul S. Successful and reproducible myocardial opacification during two-dimensional echocardiography from right heart injection of contrast. Circulation 85: 1557, 1992.

12) Keller MW, Feinstein SB, Watson DD. Successful left ventricular opacification following peripheral injection of sonicated contrast agent: an experimental evaluation. Am Heart J 114: 570, 1987.

13) Ten Cate FJ, Feinstein SB, Zwehl W, et al. Two-dimensional contrast echocardiography. II. Transpulmonary studies. J Am Coll Cardiol 3: 21, 1984.

14) Berwing K, Schlepper M. Echocardiographic imaging of the left ventricle by peripheral intravenous injection of echo contrast agent. Am Heart J 115: 399, 1988.

15) Meltzer RS, Serruys PW, McGhie J, Verbaan N, Roelandt J. Pulmonary wedge injections yielding left-sided echocardiographic contrast. Br Heart J 44: 390, 1980.

16) Smith MD, Elion JL, McClure RR, et al. Left heart opacification with peripheral venous injection of a new saccharide echo contrast agent in dogs. J Am Coll Cardiol 13: 1622, 1989.

17) Feinstein SB, Ten Cate FJ, Zwehl W, et al. Two-dimensional contrast echocardiography. I. In vitro development and quantitative analysis of echo contrast agents. J Am Coll Cardiol 3: 14, 1984.

18) Keller MW, Glasheen W, Teja K, Gear A, Kaul S. Myocardial contrast echocardiography without significant hemodynamic effects or reactive hyperemia: a major advantage in the imaging of regional myocardial perfusion. J Am Coll Cardiol 12: 1039, 1988.

19) Ohta $T$, Beppu $S$, Nakatani $S$, Miyatake K. Effect of the contrast agent and the agitation method on the size, number and stability of microbubbles: a basic experiment for the myocardial contrast study. Jpn J Med Ultrasonics 18: 32, 1991.

20) Baele PL, McMichan JC, Marsh M, Sill JC, Southorn PA. Continuous monitoring of mixed venous oxygen saturation in critically ill patients. Anesth Analg 61: 513, 1982.

21) Rah KH, Dunwiddie WC, Lower RR. A method for continuous postoperative measurement of mixed venous oxygen saturation in infants and children after open heart procedures. Anesth Analg 63: 873, 1984.

22) Rovei D, Nissen SE, Elion J, et al. Contrast echo washout curves from the left ventricle: application of basic principles of indicator-dilution theory and calculation of ejection fraction. J Am Coll Cardiol 10: 125, 1987.

23) Meltzer RS, Reisner SA. Contrast echocardiography in the Doppler color flow imaging era. J Am Coll Cardiol 13: 687, 1989.

24) Nakatani S, Imanishi T, Terasawa A, Beppu S, Nagata S, Miyatake K. Clinical application of transpulmonary contrast-enhanced Doppler technique in the assessment of severity of aortic stenosis. J Am Coll Cardiol 20: $973,1992$.

25) Terasawa A, Miyatake K, Nakatani S, Yamagishi M, Matsuda H, Beppu S. Enhancement of Doppler flow signals in the left heart by intravenous injection of sonicated albumin. J Am Coll Cardiol 21: 737, 1993.

26) Fritzsch TH, Lange L, Zimmermann I. Ultrasonogr bulletin, abstracts of the sixth symposium on echocardiology. in: A lung-crossing contrast agent for echocardiography, Kamphuisen HAD, Ed. Bohn, Scheltema \& Holkema, Inc., Utrecht/Antwerpen, 1985, p.3.

27) Walker R, Wiencek JG, Aronson S, et al. The influence of intravenous Albunex injections on pulmonary arterial pressure, gas exchange, and left ventricular peak intensity. J Am Soc Echocardiogr 5: 463, 1992. 